\title{
Variceal pressure is a strong predictor of variceal haemorrhage in patients with cirrhosis as well as in patients with non-cirrhotic portal hypertension
}

\author{
E A El Atti, F Nevens, K Bogaerts, G Verbeke, J Fevery
}

\begin{abstract}
Background-Variceal pressure is a strong predictor for a first variceal bleed in patients with cirrhosis.

Aims-To evaluate whether variceal pressure is also a determinant of the risk of a first variceal bleed in patients with noncirrhotic portal hypertension.

Methods-Variceal pressure was measured non-invasively in 25 patients with non-cirrhotic portal hypertension and large varices while receiving a stable therapeutic regimen. Factors predictive of bleeding were compared with those observed in 87 cirrhotics.

Results-The one year incidence of variceal bleeding was $32 \%(n=28)$ for the cirrhotic and $20 \%(n=5)$ for the noncirrhotic patients. There was no difference in factors predicting the risk of bleeding between the groups, except for variceal pressure. For the same level of variceal pressure, the risk of variceal bleeding was lower in patients with non-cirrhotic portal hypertension. Multiple logistic regression analysis revealed the following variables as having a significant predictive power: variceal pressure $(p=0.0001)$, red spots $(p=0.004)$, and the time interval between the first observation of the varices and the moment of variceal pressure measurement $(p=0.0046)$. For the non-cirrhotics the risk of bleeding increased with higher Child-Pugh score $(p=0.0024)$; this was not the case for the cirrhotic patients $(\mathrm{p}=0.9521)$.
\end{abstract}

Department of Liver and Pancreatic Diseases, University Hospital, Leuven, Belgium

E A El Atti

F Nevens

J Fevery

Biostatistical Center for Clinical Trials, University Hospital, Leuven, Belgium

K Bogaerts

G Verbeke

Correspondence to: Dr F Nevens, Division of Liver and Pancreatic

Diseases, University Hospital

Gasthuisberg, B-3000

Leuven, Belgium.

Accepted for publication 27 April 1999

Portal hypertension is a major complicatio chronic liver disease. A clinical consequence, bleeding from ruptured oesophageal varices is one of the major causes of death in portal hypertensive patients. ${ }^{12}$ It has been established that in alcoholic cirrhosis the wedged hepatic venous pressure (WHVP) is nearly identical to the portal vein pressure. ${ }^{3}$ A reduction of the hepatic venous pressure gradient (HVPG) to less than $12 \mathrm{~mm} \mathrm{Hg}$ protects against an episode of bleeding in cirrhotic patients ${ }^{4}$ and it has been claimed that a reduction of HVPG is correlated with a lower risk of variceal rebleeding. ${ }^{5}$ As such this measurement has been propagated for use in the follow up of cirrhotic patients under pharmacotherapy in the primary and secondary prevention of variceal bleeding. ${ }^{6}$

Portal hypertension can, however, also result from non-cirrhotic causes. In most of these, the pathogenesis is related to an obliterative venopathy of the extrahepatic or intrahepatic portal vein. ${ }^{78}$ In the West, portal vein thrombosis is the most common cause of non-cirrhotic portal hypertension ${ }^{9}$; worldwide, hepatic schistosomiasis is one of the world's most prevalent chronic liver diseases. ${ }^{10}{ }^{11}$ Rupture of oesophageal varices is common in this group of disorders and may be the first clinical manifestation of the disease..$^{12}$ In these conditions WHVP will underestimate portal pressure. ${ }^{14} 15$ In patients with non-cirrhotic portal hypertension, variceal pressure bears an excellent linear relation with portal pressure. ${ }^{15}$ Variceal pressure can be measured non-invasively with an endoscopic probe. ${ }^{16-19}$ The level of variceal pressure is a strong predictor for a first variceal bleed in patients with cirrhosis. ${ }^{20}$ In patients with non-cirrhotic portal hypertension, variceal pressure seems therefore an attractive parameter to assess the risk of variceal bleeding. Furthermore, the incidence and the risk factors of variceal bleeding in non-cirrhotic portal hypertensive patients have not been fully evaluated.

The aim of the present study was to evaluate the risk factors of variceal haemorrhage, together with the role of variceal pressure for the prediction of a first variceal bleed in patients with non-cirrhotic portal hypertension.

\section{Patients and methods}

The study population consisted of 112 patients with large varices: 87 cirrhotic patients previously reported ${ }^{20}$ and 25 consecutive patients with non-cirrhotic portal hypertension in whom variceal pressure was measured during their work up. The variceal pressure performed when patients were under a stable drug regimen (such as propranolol) for at least one
Abbreviations used in this paper: HVPG, hepatic venous pressure gradient; WHVP, wedged hepatic venous pressure. 
Table 1 Univariate analysis of different parameters at the time of inclusion, investigated for their role in the risk of variceal bleeding

\begin{tabular}{|c|c|c|c|c|c|c|}
\hline & \multicolumn{2}{|l|}{ Cirrhotics } & \multicolumn{2}{|l|}{ Non-cirrhotics } & \multicolumn{2}{|l|}{ p Value } \\
\hline & Bleeders $(n=28)$ & Non-bleeders $(n=59)$ & Bleeders $(n=5)$ & Non-bleeders $(n=20)$ & $\begin{array}{l}\text { Risk of bleeding for } \\
\text { all patients }(n=112)\end{array}$ & $\begin{array}{l}\text { Difference between } \\
\text { both groups }\end{array}$ \\
\hline Age (years) & $61(11.3)(34-78)$ & $58.1(12.4)(24-76)$ & $51.6(11.7)(35-65)$ & $43.5(18.4)(18-75)$ & 0.189 & \\
\hline \multicolumn{7}{|c|}{ 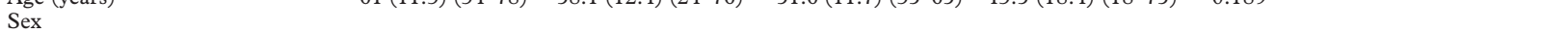 } \\
\hline Male & $19(67.9 \%)$ & $39(66.1 \%)$ & $1(20 \%)$ & $5(25 \%)$ & 0.9522 & \\
\hline Female & $9(32.1 \%)$ & $20(33.9 \%)$ & $4(80 \%)$ & $15(75 \%)$ & & \\
\hline Child-Pugh score (points) & $8(2.6)(5-13)$ & $7.5(2.4)(5-14)$ & $7.8(2)(6-10)$ & $6.1(1.9)(5-13)$ & 0.181 & \\
\hline \multirow[t]{2}{*}{ Prothrombin time $(\%)$} & $57.8(19.2)$ & $58.7(20.7)$ & $66.6(22.1)$ & $70.4(25.6)$ & & \\
\hline & $(24.3-100)$ & $(21-100)$ & $(41.8-87)$ & $(13.7-100)$ & 0.7497 & \\
\hline \multirow[t]{2}{*}{ Albumin (mg/dl) } & $3.46(0.51)$ & $3.66(0.56)$ & $3.31(0.43)$ & $3.87(0.53)$ & & \\
\hline & $(2.48-4.78)$ & $(2.36-4.78)$ & $(2.6-3.67)$ & $(2.34-4.55)$ & $0.0253^{\star}$ & 0.2796 \\
\hline \multirow[t]{2}{*}{ Bilirubin (mg/dl) } & $2.75(2.51)$ & $2.47(2.2)$ & $1.8(1.9)$ & & & \\
\hline & $(0.7-9.26)$ & $(0.54-13.6)$ & $(0.4-4.99)$ & $1.3(1.2)(0.43-6.1)$ & 0.7497 & \\
\hline \multicolumn{7}{|l|}{ Encephalopathy } \\
\hline Present & $10(35.7 \%)$ & $14(23.7 \%)$ & $0(0 \%)$ & $0(0 \%)$ & 0.5075 & \\
\hline Absent & $18(64.3 \%)$ & $45(76.3 \%)$ & $5(100 \%)$ & $20(100 \%)$ & & \\
\hline \multicolumn{7}{|l|}{ Ascites } \\
\hline Present & $15(53.6 \%)$ & $18(30.5 \%)$ & $4(80 \%)$ & $3(15 \%)$ & $0.003^{\star}$ & 0.1147 \\
\hline Absent & $13(46.4 \%)$ & $41(69.5 \%)$ & $1(20 \%)$ & $17(85 \%)$ & & \\
\hline Size of varices $(\mathrm{mm})$ & $7.8(1.7)(5-10)$ & $5.4(2.1)(3-10)$ & $7.6(2.9)(4-10)$ & $5.4(2.4)(3-10)$ & $0.0001^{\star}$ & 0.2156 \\
\hline \multicolumn{7}{|l|}{ Red colour signs } \\
\hline Yes & $19(67.9 \%)$ & $16(27.1 \%)$ & $3(60 \%)$ & $8(40 \%)$ & $0.0005^{\star}$ & 0.4157 \\
\hline No & $9(32.1 \%)$ & $43(72.9 \%)$ & $2(40 \%)$ & $12(60 \%)$ & & \\
\hline \multirow[t]{2}{*}{ NIEC index (points) } & $37.3(7.9)$ & $28.7(6.6)$ & $34.7(5.3)$ & $26.8(6.3)$ & & \\
\hline & $(22.7-54.4)$ & $(18.4-44.7)$ & $(27.3-40.1)$ & $(18.4-38.2)$ & $0.0001^{\star}$ & 0.4953 \\
\hline \multirow[t]{2}{*}{ Variceal pressure $(\mathrm{mm} \mathrm{Hg})$} & $17.7(2.9)$ & & $20.1(6.3)$ & $15.5(4.7)$ & & \\
\hline & $(13.8-24.8)$ & $13.5(2.6)(8-21.3)$ & $(12.4-30)$ & $(7.3-23.6)$ & & $0.0412^{\star}$ \\
\hline Known time since varices (months) & $10.5(1-59)$ & $23(1-204)$ & $46(1-106)$ & $48(5-396)$ & $0.0135^{\star}$ & 0.1274 \\
\hline
\end{tabular}

Results expressed as mean (SD) (range)

month, was taken as baseline. The medication regimen was kept unchanged during the whole study period and the patients were followed up for one year.

The cirrhotic group consisted of 58 men and 29 women with a mean age of 59 (12) years (range 24-78). Cirrhosis was alcoholic in origin in 32 patients, due to hepatitis $\mathrm{C}$ in 18 , hepatitis $\mathrm{B}$ in 8 , autoimmune hepatitis in three, and was cryptogenic in 12 patients. Nine patients suffered from primary biliary cirrhosis, three from primary sclerosing cholangitis, one had haemochromatosis, and one had Wilson's disease.

In the non-cirrhotic group there were six men and 19 women with a mean age of 46 (17) years (range 18-75). The aetiology was portal vein thrombosis in 15 patients, incomplete septal cirrhosis in four, nodular regenerative hyperplasia in three, and hepatoportal sclerosis in three patients.

The severity of the liver disease was graded according to the Child-Pugh criteria: 34 (39\%) cirrhotic and $17(68 \%)$ non-cirrhotic patients belonged to Child's class A, 37 (42\%) cirrhotic and five $(20 \%)$ non-cirrhotic patients to class $\mathrm{B}$, and $16(18 \%)$ cirrhotic and three (12\%) non-cirrhotic patients to class $\mathrm{C}$. Thirty three $(38 \%)$ in the cirrhotic group and seven $(28 \%)$ in the non-cirrhotic group had ascites. The interval between the endoscopic diagnosis of varices and the start of the study was 15 months (range 1-204) in the cirrhotic group and 47 months (range 1-396) in the noncirrhotic group.

Measurements of variceal pressure were performed at endoscopy, using a pressure sensitive gauge which was adapted in our unit. ${ }^{16-19}$ All patients received premedication with 2.5-7.5 $\mathrm{mg}$ diazepam and $40 \mathrm{mg} n$-butylscopolamine intravenously. The system uses a small hemispheric gauge attached to the distal end of an endoscope which is applied over an oesophageal varix. The recording of variceal pressure was considered satisfactory when fine venous fluctuations at a stable level, superimposed on the respiratory cycle, were recognised in the absence of pronounced luminal contraction, and when free oesophageal pressure did not exceed $5 \mathrm{~mm} \mathrm{Hg}$. Pressures were calculated from the mean of the upper and lower level of the fluctuations. Variceal pressure was considered as the difference between the capsule pressure and the free oesophageal luminal pressure. The mean of at least five satisfactory measurement periods was used to define variceal pressure.

After variceal pressure measurement, the size of the varix was estimated during gentle insufflation of the oesophagus and in the absence of peristaltic waves, by comparing the varix with the internal and external size of the pressure gauge ( $5 \mathrm{~mm}$ and $7.5 \mathrm{~mm}$, respectively). The maximal diameter of the varices was recorded and the red colour signs were noted.

Table 1 presents other parameters investigated for their value in the prediction of a first bleeding; the NIEC classification was calculated as previously published. ${ }^{21}$

The endpoint of the study was the presence or absence of a variceal bleed within one year. Bleeding was defined as any episode of variceal bleeding evidenced by haematemesis or meleana during follow up. Whenever bleeding occurred, patients were admitted to the hospital and the source of bleeding was confirmed by endoscopy. Variceal bleeding was diagnosed if there was an active bleeding varix or blood clots on the varices, or if no other cause of bleeding could be found. Death was considered to be related to variceal bleeding if it occurred within six weeks after the onset of bleeding. 


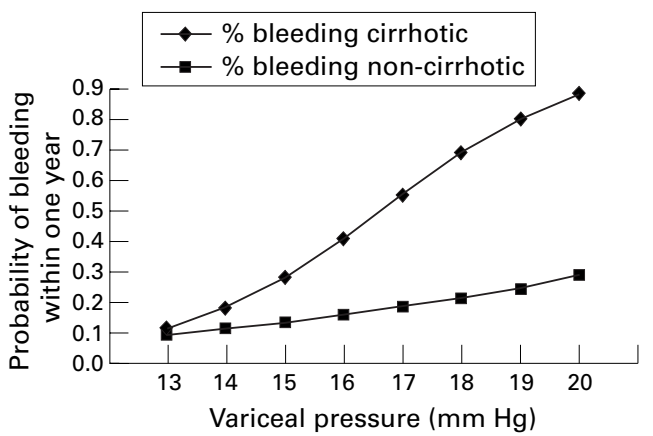

Figure 1 Relation of variceal pressure to the risk of variceal haemorrhage.

\section{STATISTICAL ANALYSIS}

The relation between bleeding and several covariates was described by means of a logistic regression (simple and multiple). It was allowed that the effect of a certain variable could be different for cirrhotic and noncirrhotic patients. In the multiple analysis, there was a correction for age and sex. The level of significance was set at 0.05 .

\section{Results}

Variceal bleeding occurred within one year in 28 patients $(32 \%)$ with cirrhosis and in five patients $(20 \%)$ with non-cirrhotic portal hypertension. The one year mortality was $16 \%$ $(n=14)$ in the cirrhotic group; 36\% $(n=5)$ died from a variceal bleeding. In the non-cirrhotics the one year mortality was $8 \%(n=2)$; only one patient died from variceal bleeding.

The simple analysis revealed that variables predictive of the first variceal bleed in the whole study group were the level of variceal pressure, the variceal size $(p=0.0001)$, the presence of red spots $(p=0.0005)$, the NIEC score $(p=0.0001)$, the presence of ascites $(p=0.003)$, and the time interval between diagnosis of the varices and the start of the study $(p=0.0135)$. The effect of these variables on the risk of bleeding did not differ between the cirrhotic and non-cirrhotic patients, except for the variceal pressure level $(p=0.00412)$. For the same level of variceal pressure the risk of bleeding was lower in patients with noncirrhotic portal hypertension than in patients with cirrhosis (see fig 1).

With the results obtained from the simple analysis, a multiple logistic regression model was created which revealed that after correction for age and sex, variceal pressure $(p=0.0001)$, red spots $(p=0.004)$, the time interval between first observation of varices and the moment of variceal pressure measurement $(p=0.0046)$, and the Child-Pugh score were independent predictors of the risk of a first variceal bleeding in both groups.

For the non-cirrhotics the risk of bleeding increased with higher Child-Pugh score $(p=0.0084)$; this was not the case for the cirrhotic patients $(\mathrm{p}=0.9521)$.

\section{Discussion}

In general, a variceal haemorrhage is better tolerated in non-cirrhotic portal hypertensive patients than in cirrhotics. ${ }^{1022}$ Nevertheless, the mortality rate due to a first episode of bleeding may reach $25 \%$ in patients with schistosomal hepatic fibrosis, ${ }^{23}$ and multiple bleeding episodes which extend over years are characteristic for this group of disorders. ${ }^{23}$

Non-cirrhotic portal hypertension is prevalent in countries where well equipped hospitals with the possibility of unlimited blood transfusion are not always available. Therefore prognostic information to identify patients at risk of bleeding, who might be candidates for prophylactic treatment is of interest for these patients. Oesophageal devascularisation with splenectomy, portosystemic shunting procedures, ${ }^{24}$ injection sclerotherapy, and propranolol have been proposed as therapy in the primary and secondary prevention for patients with noncirrhotic portal hypertension. ${ }^{23}{ }^{25}$

In patients with alcoholic cirrhosis, WHVP reflects portal pressure. ${ }^{3}$ In patients with presinusoidal and prehepatic portal hypertension, measurement of HVPG fails to indicate high portal pressure. Therefore measurements of HVPG cannot be used as a parameter to assess the degree of portal hypertension in these conditions. ${ }^{14}$ Sarin and colleagues ${ }^{15}$ reported that variceal pressure closely correlates with intrasplenic pressure and as such with portal pressure in non-cirrhotic patients with portal hypertension. We and others showed a close relation with variceal pressure values obtained by needle puncture with the values obtained by the non-invasive device. ${ }^{18}{ }^{26}$ The major advantage of variceal pressure measurements by using a hemispheric pneumatic pressure gauge is that it can be used at routine endoscopy in patients who have not previously bled from their varices, without the risk of precipitating variceal haemorrhage. With the latter technique we showed in cirrhotics that variceal pressure is a major determinant in the development of a variceal bleed. ${ }^{20}$

The present study shows that the close relation which exists between the pressure in the varices and imminent variceal bleeding in cirrhotics also exists in non-cirrhotic patients. However, for the same level of variceal pressure the risk of bleeding is lower in non-cirrhotic patients with portal hypertension than in cirrhotics, which further confirms that besides haemodynamic parameters, other factors, such as the liver function capacity, may play a role in the development of variceal haemorrhage. ${ }^{21}$ In the present study the liver function, as assessed by the Child-Pugh score was significantly related to the risk of bleeding in both groups. In non-cirrhotic patients, however, the risk of variceal bleeding was more pronounced in the case of decompensation than in cirrhotic patients. This might be explained by the fact that when patients with non-cirrhotic portal hypertension finally develop tense ascites and liver function disturbances, they are in a more advanced end stage of their disease. This was reflected in our series by the fact that some of these patients subsequently had to undergo a liver transplantation despite the histological absence of established cirrhosis.

In conclusion, in contrast to HVPG, variceal pressure is a major parameter for prediction of 
a first variceal bleed for all patients with oesophageal varices independent of the aetiology of portal hypertension. The present study revealed that non-cirrhotics bled at a higher level of variceal pressure than cirrhotics; but the risk of variceal bleeding increases more in non-cirrhotic patients with advancing disease, especially when ascites becomes an important symptom. This study further confirms that variceal pressure measurements are important as a diagnostic method in the follow up of all patients with portal hypertension.

1 Nevens F, Broeckaert L, Rutgeerts P, et al. The long-term morbidity and mortality rate in a cohort of patients with liver cirrhosis and oesophageal varices. Hepatogastroenterolliver cirrhosis and oesop
ogy 1995;42:979-84.

2 Burroughs AK. The natural history of varices. 7 Hepatol 1993;17(suppl 2):S10-13.

3 Boyer TD, Tiger DR, Horisawa M, et al. Direct transhepatic measurement of portal vein pressure using a thin needle. Comparison with wedged hepatic vein pressure. Gastroenterology 1977:72:584-9.

4 Groszmann RJ, Bosch J, Grace ND, et al. Hemodynamic events in a prospective randomized trial of propranolol versus placebo in the prevention of a first variceal hemorrhage. Gastroenterology 1990;99:1401-7.

5 Feu F, Garcia-Pagan JC, Bosch J, et al. Relation between portal pressure response to pharmacotherapy and risk of recurrent variceal haemorrhage in patients with cirrhosis. Lancet 1995;346:1051-9.

6 Armonis A, Patch D, Burroughs A. Hepatic venous pressure measurement: an old test as a new prognostic marker in cirrhosis? Hepatology 1997;25:245-8.

7 Nevens F, Staessen D, Sciot R, et al. Clinical aspects of incomplete septal cirrhosis in comparison with macronodular cirrhosis. Gastroentrology 1994;106:459-63.

8 Valla D, Casadevall N, Huisse MG, et al. Aetiology of portal vein thrombosis in adults. A prospective evaluation of vein thrombosis in adults. A prospective evaluation of
primary myeloproliferative disorders. Gastroenterology primary myelop

9 Sherlock S. Noncirrhotic extrahepatic and intrahepatic portal hypertension. Semin Liver Dis 1982;2:202-10.

10 Da Silva LC, Carrilho FJ. Hepatosplenic schistosomiasis. Pathophysiology and treatment. Gastroenterol Clin North Am 1992;21:163-77.
11 Dunn MA, Kamel R. Hepatic schistosomiasis. Hepatology 1981;1:653-61.

12 Sarin SK. Non-cirrhotic portal fibrosis. Gut 1989;30:40615

13 Lurgen J, Hashimoto E, Obata H, et al. Idiopathic portal hypertension. Hepatology 1993;17:1157-62.

14 Pomier-Layrargues G, Kusieleewicz D, Willems B, et al. Presinusoidal portal hypertension in non-alcoholic cirrhosis. Hepatology 1985;5:415-18.

15 Sarin SK, Sethi KK, Nanda R. Measurement and correlation of wedged hepatic, intrahepatic, intrasplenic and intravariceal pressures in patients with cirrhosis of liver and non-cirrhotic portal fibrosis. Gut 1987;28:260-6.

16 Rigau J, Bosch J, Bordas J, et al. Endoscopic measurement of variceal pressure in cirrhosis: correlation with portal variceal pressure in cirrhosis: correlation with portal
pressure and variceal hemorrhage. Gastroenterology 1989; pressure and $873-80$.

17 Nevens F, Van Steenbergen W, Yap SH, et al. Assessment of variceal pressure by continuous non-invasive endoscopic registration: a placebo controlled evaluation of the effect of terlipressin and octreotide. Gut 1996;38:129-34.

18 Nevens F, Fevery J. Measurement of variceal pressure and its clinical implications. Scand $\mathcal{F}$ Gastroenterol 1994; (suppl 207):6-10.

19 Nevens F, Sprengers D, Fevery J. The effect of different doses of a bolus injection of somatostatin combined with a slow infusion on transmural oesophageal variceal pressure in patients with cirrhosis. F Hepatol 1994;20:27-31.

20 Nevens F, Bustami R, Scheys I, et al. Variceal pressure is a factor predicting the risk of a first variceal bleeding: a prospective cohort study in cirrhotic patients. Hepatology

21 The North Italian Endoscopic Club for the Study and Treatment of Oesophageal Varices. Prediction of the first variceal hemorrhage in patients with cirrhosis of the liver and oesophageal varices. A prospective multicenter study. N Engl F Med 1988;319:983-9.

22 Lebrec D, Benhmou J. Noncirrhotic intrahepatic portal hypertension. Semin Liver Dis 1986;6:332-40.

23 Bessa SM, Helmy I. Injection sclerotherapy for oesophageal varices caused by schistosomal hepatic fibrosis. Surgery 1985;97:164-8

24 Raia S, Da Silva LC, Gayotto LCC, et al. Portal hypertension in schistosomiasis: a long-term follow-up of a randomized trial comparing three types of surgery. Hepatology 1994;20:398-403.

25 Braillon A, Moreau R, Hadengue A, et al. Hyperkinetic circulatory syndrome in patients with presinusoidal portal hypertension. Effect of propranolol. F Hepatol 1989;9:31218

26 Lebrec D. Methods to evaluate portal hypertension. Gastroenterol Clin North Am 1992;21:41-59. 